Editorial

\title{
Acknowledgement to Reviewers of Catalysts in 2017
}

\section{Catalysts Editorial Office}

MDPI AG, St. Alban-Anlage 66, 4052 Basel, Switzerland

Published: 11 January 2018

Peer review is an essential part in the publication process, ensuring that Catalysts maintains high quality standards for its published papers. In 2017, a total of 385 papers were published in the journal. Thanks to the cooperation of our reviewers, the median time to first decision was 14 days and the median time to publication was 40 days. The editors would like to express their sincere gratitude to the following reviewers for their time and dedication in 2017:

\begin{tabular}{|c|c|c|}
\hline Abanades, Stéphane & Hara, Michikazu & Oh, Jae-Min \\
\hline Abatzoglou, Nicolas & Härelind, Hanna & Oh, Kyungsoo \\
\hline Abdala, Paula Macarena & Hargreaves, Justin & Ohtani, Bunsho \\
\hline Abdelwahed, Sameh & Hartl, František & Okumura, Hideyuki \\
\hline Abo, Rudy K. & Haruki, Mitsuru & Ollis, David \\
\hline Abu-Reziq, Raed & Hashimoto, Makoto & Olsbye, Unni \\
\hline Acha, Victor & Haunschild, Robin & Olthuis, W. \\
\hline Adiya, Zisg & Hausinger, Robert & Omata, Kohji \\
\hline Agrios, Alexander & Hayakawa, Yasuhiro & Orian, Laura \\
\hline Åkermark, Björn & Heo, Jinseok & Osakada, Kohtaro \\
\hline Akhavan, Omid & Hernández, Simelys & Osatiashtiani, Amin \\
\hline Akizawa, Toshifumi & Hernández-Garrido, J. C. & Oshiki, Toshiyuki \\
\hline Alayoglu, Selim & Herrera Delgado, María Concepción & Pablos, Cristina \\
\hline Albani, Davide & Herres-Pawlis, Sonja & Paganini, Maria Cristina \\
\hline Alba-Rubio, Ana C. & Hideki, Kurokawa & Pagan-Torres, Yomaira J. \\
\hline Albert, Machoke & Higashida, Suguru & Paik, Ungyu \\
\hline Albini, Angelo & Higuchi, Eiji & Palmisano, Leonardo \\
\hline Albo, Jonathan & Hikawa, Hidemasa & Palomares, Antonio Eduardo \\
\hline Alegre, Cinthia & Hinds, Gareth & Panomsuwan, Gasidit \\
\hline Alessandri, Ivano & Hippler, Rainer & Pap, Zsolt \\
\hline Alexandr V., Talyzin & Hirao, Hajime & Papadopoulou, Christina \\
\hline Almquist, Catherine & Hisada, Kenji & Papamichael, Emmanuel \\
\hline Alphonse, Pierre & Hofmann, Jan Philipp & Paraskevopoulou, Patrina \\
\hline Altomare, Marco & Hollenstein, Marcel & Park, Gu-Gon \\
\hline Amadelli, Rossano & Hong, Suck Won & Park, Young-Kwon \\
\hline Amos, Ruth I.J. & Horinek, Dominik & Parlett, Christopher \\
\hline Amreddy, Narsireddy & Hornung, Andreas & Parmeggiani, Fabio \\
\hline Amutio, Maider & Hosseini, Mahsa & Pasini, Dario \\
\hline Andreescu, Silvana & Hou, Jingwei & Patsios, Sotiris I. \\
\hline
\end{tabular}


Antoniotti, Sylvain Apfel, Ulf-Peter

Arai, Takayoshi

Aranzabal, Asier

Arena, Francesco

Argia, Katsuhiko

Armandi, Marco

Armbruster, Udo

Arvapally, Ravi Kumar

Ashekuzzaman, S.M.

Asthagiri, Aravind

Atia, Hanan

Atsonios, Konstantinos

Audette, Gerald F

Axet, M. Rosa

Ayers, Paul

Bae, Byeong-Soo

Bae, Changdeuck

Bae, Joongmyeon

Baeza, Alejandro

Baglio, Vincenzo

Balcar, Hynek

Ballesté, Ruben Mas

Barla, Thirupathi

Bauer, Eike

Bavaro, Teodora

Beall, Gary W.

Beard, James D.

Bechelany, Mikhael

Bell, Carl-Martin

Bellardita, Marianna

Benaglia, Maurizio

Bensaid, S.

Bentrup, Ursula

Bergbreiter, David

Bharadwaj, Vivek

Bhavsar, Saurabh

Biasi, Pierdomenico

Bobicki, Erin R.

Boehr, David D

Bogel-Łukasik, Rafał

Bolivar, Juan M.

Bonifácio, Vasco
Hoyos, Pilar

Hsu, Cheng-che

$\mathrm{Hu}$, Anming

$\mathrm{Hu}$, Maocong

Huang, Chih-Ching

Huang, Hao

Hudson, Reuben H.

Hui, K. N.

Huirache-Acuna, R.

Husson, Jérôme

Hwang, WanSik

Ibrahim, Hussameldin

Ierapetritou, Marianthi

Iglesias, Marta

Iida, Hajime

Ikenaga, Naoki

Iloeje, Chukwunwike

Imai, Hiroyuki

Impellizzeri, Giuliana

Infantes Molina, Antonia

Iniesta Valcarcel, Jesus

Ioannides, Theophilos

Ipsakis, Dimitris

Ishitani, Haruro

Ito, Jun-ichi

Ito, Shingo

Itsuno, Shinichi

Ivanova, Svetlana

Izzo, Viviana

Jackson, Michael A.

Jacobsen, Elisabeth Egholm

Jagdale, Pravin

Jain, Ankur

James G., Dale

Janesko, Benjamin G.

Jang, Ho Won

Janiak, Christoph

Jariwala, Deep

Jena, Umakanta

Jeon, Seokwoo

Jeong, Lak Shin

Jepsen, Julian

Jess, Andreas
Pattabiraman, Mahesh

Patterson, Howard H.

Pavlidis, Ioannis

Pawelec, Barbara

Pedrosa, Rafael

Pellegrino, Francesco

Pellejero, Ismael

Pellis, Alessandro

Peng, Zhili

Pereira, Mariette M.

Pérez, Esther Enríquez

Periana, Roy A.

Pesci, Federico M

Peter, Ngene

Peters, Thijs

Phuong, Nguyen-Tri

Pia, Giorgio

Piccirillo, Clara

Piermatti, Oriana

Pietschmig, Prof. Dr. Rudolf

Pigge, F. Christopher

Pignataro, Luca

Pindi, Suresh

Pires, E.

Pirola, Carlo

Pita, Marcos

Pizarro, Patricia

Pizzano, Antonio

Plakas, Konstantinos

Plasseraud, Laurent

Pleixats, Roser

Poater, Albert

Poelman, Hilde

Politano, Antonio

Polverino, Pierpaolo

Pourceau, Gwladys

Prata, José V.

Prati, Laura

Predoi, Daniela

Protasova, Lidia

Puccini, Monica

Puga, Alberto V.

Qiang, Zhe 
Bordes-Richard, Elisabeth

Bosso, Luciano

Boury, Bruno

Boyd, Joel

Braeuninger, Philipp

Brankovic, Stanko

Brenna, Elisabetta

Brillard, Alain

Brinkmann, Torsten

Brito, Manuel E.

Brookhart, Maurice

Bueno-López, Agustín

Buonocore, Francesco

Cacciato, Giuseppe

Cadierno, Victorio

Camarillo, Rafael

Camblor, Miguel A.

Camera-Roda, Giovanni

Campbell, Michael

Cámpora, Juan

Candeias, Nuno R.

Canivet, Jerome

Caporali, Stefano

Carabineiro, Sónia A.C.

Carbonnier, Benjamin

Cardoso, David

Carleer, Robert

Carlsson, Per-Anders

Caselli, Alessandro

Castanheiro, José

Castoldi, Lidia

Cavazza, Christine

Cecilia, Juan Antonio

Celorrio, Veronica

Centeno, Miguel Angel

Cesano, Federico

Chaikittisilp, Watcharop

Chatel, Gregory

Chavez, Ferman

Chen, Chiing-Chang

Chen, Luwei

Chen, Peirong

Chen, Shih-Yuan

$$
\text { Ji, Li }
$$

Jitianu, Mihaela

Jo, Wan-Kuen

Jo, Young Min

João Pires, Da Silva

Jooss, Christian

Joseph, Babu

Jradi, Khalil

Ju, Myung-Jong

Kabanos, Themistoklis

Kadakowa, Prof. Jun-ichi

Kakinuma, Katsuyoshi

Kako, Tetsuya

Kalantar-Zadeh, Kourosh

Kalevaru, Narayana

Kalogiannis, Konstantinos G.

Kamada, Kai

Kan, Eunsung

Kanda, Yasuharu

Kaneda, Kiyotomi

Kanel, Sushil R.

Kang, Youngsoo

Kanna, Machi

Kataev, Evgeny

Kawato, Yuji

Kayaki, Yoshihito

Keul, Helmut

Khan, Muhammad

Khurshid, Zohaib

Kim, Dong Hyun

Kim, Hasuck

Kim, Hoon

Kim, Hyun-Ha

Kim, Jae Chang

Kim, Jae-Kon

Kim, Jin Young

Kim, Jinho

Kim, Jungbae

Kim, Kwang

Kim, Sang Hoon

Kim, Seonah

Kim, Sooyoung

Kim, T. Doohun
Rackemann, Darryn

Raffelt, Klaus

Raillard, Cécile

Ramesh, Sreerangappa

Ramis, Gianguido

Rana, Dipak

Raspoli, Anna

Ravelli, Davide

Razavi, Pedram

Reddy, Amaranatha

Reddy, M.V.

Reetz, Manfred T.

Rehm, Thomas H

Renz, Michael

Renzi, Polyssena

Reyes-Labarta, J. A.

Richards, Christopher J.

Richards, Nigel

Rimoldi, Luca

Rizo, Rubén

Rizzi, Gian Andrea

Rodenburg, Lisa

Rodrigues, Alírio

Rodríguez, Jesús Álvarez

Rodriguez-Castellon, Enrique

Rodriguez-Couto, Susana

Roduner, Emil

Roglans, Anna

Rojas, Sergio

Román-Martínez, M.C.

Rose, Marcus

Rtimi, Sami

Ruiz, Edelmira Valero

Salahub, Dennis

Salguero, Francisco J. Romero

Samec, Joseph

Sánchez, Pedro Castaño

Sanjust, Enrico

Santini, Carlo

Santos, Diogo

Saoud, Khaled

Sarkar, Dhruba

Sasai, Yasushi 


Chen, Zhichao
Chen, Zhijie
Cheng, Kong-Wei
Cheng, Qing-Qing
Cheng, Shouyun
Cheng, Tao
Cherepanov, Pavel
Cherevan, Alexey
Cherkaoui Malki, Mustapha
Chiarello, Gian Luca
Chinchilla, Rafael
Chmely, Stephen C
Cho, In Sun
Choi, Nag Jung
Choinopoulos, Ioannis
Chu, Shidong
Chuang, Po-Ya Abel
Chung, Hoyong
Ciardelli, Francesco
Cirillo, Giuseppe
Claver, Carmen
Clomburg, James
Coul

Cole, Matthew Thomas

Contreras, Sandra

Cooper, Arthur J.L.

Costa Pessela João, Benevides

Costas, Miquel

Crabtree, Robert $\mathrm{H}$.

Creaser, Derek

Cristiani, Pierangela

Croatt, Mitchell P.

Crocker, Mark

D'Anna, Francesca

Da Silva, Rafael

Dacquin, Jean-Phillipe

Dagdeviren, Canan

Dal Santo, Vladimiro

Dallinger, Doris

Damkaci, Fehmi

Dasgupta, Srimoyee

Davis, Burtron

De Bellefon, Claude

De Jonghe, Steven
Kim, Young Dok

Kim, Yu Kwon

Kirillov, Alexander M.

Kiss, János

Kitahara, Tatsumi

Klose, Holger

Knölker, Hans-Joachim

Knoshaug, Eric

Ko, Kyoung Chul

Kohtani, Shigeru

Kolar, Praveen

Koller, Martin

Konsolakis, Michalis

Koo, Bon

Kooyman, P. J.

Kopylovich, Maximilian

Kordulis, Christos

Kosinov, Nikolay

Kowalska, Ewa

Krewald, Vera

Krivtsov, Igor

Kröcher, Oliver

$\mathrm{Ku}$, Seockmo

Kubota, Yoshihiro

Kukla, Maija

Kunjukunju, Sangeetha

Kurniawan, Winarto

Kuroiwa, Takashi

Kwon, Seong Jung

La Parola, Valeria

Ladero, Miguel

Laguna, O.H.

Laguna, Oscar Hernando

Lai, Qinghua

Lamas, María Isabel

Lammertink, Rob G. H.

Landete, José Maria

Landi, Gianluca

Lange, Heiko

Lao, Ka Un

Lara-Sanchez, Agustin

LaRue, Jerry

Lauterbach, Lars
Sasson, Yoel

Sato, Fumiya

Sato, Hirotaka

Sato, Kazuhiko

Satokawa, Shigeo

Scarso, Alessandro

Schaidle, Josh

Scheu, Christina

Schneider, Raphaël

Schwank, Johannes

Schwarze, Michael

Scirè, Salvatore

Sebastián, David

Seifert, Gotthard

Selli, Elena

Sels, Bert F.

Semagina, Natalia

Semeril, David

Senftle, Thomas

Seo, Dong-Kyun

Seo, Hyo Jin

Sequeira, César

Shanmugan, Sangaraju

Shatalov, Anatoly

Shimada, Toshihiro

Shin, Chae-Ho

Shiono, Takeshi

Shokouhimehr, Mohammadreza

Sicilia, Emila

Siddiki, S.M.A. Hakim

Sieber, Volker

Signoretto, Michela

Silvério, Sara C.

Simmons, Blake

Simons, Thomas

Singh, Gurvinder

Skoda-Földes, Rita

Skou, Eivind Morten

Smirniotis, Panagiotis G.

Smith, Kevin

Snyder, Nicole

Sobhana, Liji

Solans-Monfort, Xavier 


\begin{tabular}{|c|c|c|}
\hline De Lucas Consuengra, Antonio & Lavandera, Iván & Solsona, Benjamín \\
\hline Deganello, Francesca & Leadbeater, Nicholas & Son, Seung Uk \\
\hline Dekel, Dario R. & Leclerc, Corey & Song, Xiaobo \\
\hline Del Hierro, Isabel & Lecomte, Philippe & Sotiropoulos, Sotirios \\
\hline Delahay, Gerard & Lee, Adam & Spasiano, Danilo \\
\hline Delaude, Lionel & Lee, Bun Yeoul & Srivastava, Varsha \\
\hline Delbecq, Frederic & Lee, Hon Man & Stanley, Jessica \\
\hline Dell'Anna, Maria Michela & Lee, Jae Sung & Stefano, Stefano Di \\
\hline Della Ca', Nicola & Lee, Sunwoo & Stelmachowski, Paweł \\
\hline Della Torre, Augusto & Lee, Yong Rok & Stenstrøm, Stenstrøm \\
\hline Deng, Yelin & Lefebvre, Frederic & Stichnothe, Heinz \\
\hline Desmet, Tom & Lefebvre, Frédéric & Storaro, Loretta \\
\hline Dewi, Mewahyu & Léger, Bastien & Strini, Alberto \\
\hline Di Bartolomeo, Antonio & Lei, Weiwei & Strunk, Jennifer \\
\hline Di Credico, Barbara & Leistner, K.C. & $\mathrm{Su}$, Chunming \\
\hline Di Natale, Francesco & Lemonidou, Angeliki & Sugai, Takeshi \\
\hline Di Noto, Vito & Lenarda, Maurizio & Suganuma, Satoshi \\
\hline Di Sarli, Valeria & Leus, Karen & Sugiyama, Shigeru \\
\hline Díaz De Tuesta, Jose Luis & Levesque, Pierre L. & Suib, Steven \\
\hline Diaz, Jose Antonio & Leyva-Pérez, Antonio & Sulman, Esther \\
\hline Diego, Luna Martínez & Lianos, Panagiotis & Sun, Hongyu \\
\hline Diesendruck, Charles E. & Licini, Giulia & Sun, Luyi \\
\hline Díez, David & Liguori, Angelo & SUN, Meng \\
\hline Díez-Ramírez, J. & Likodimos, Vlassios & Sutherland, John B. \\
\hline Dijkstra, Bauke W. & Lim, Tak-hyoung & Suzuka, Toshimasa \\
\hline Dimakis, Nicholas & Lim, Yee Fun & Suzuki, Masahito \\
\hline Dimitratos, Nikolaos & Lin, Yuan-Chung & Suzuki, Yasuhiro \\
\hline Ding, Qingbao & Lin, Yuyuan & Swadźba-Kwaśny, Małgorzata \\
\hline Dismukes, G. Charles & Linares, $\mathrm{N}$. & Symes, Mark D. \\
\hline Do, Trong-On & Ling, Yong-Chien & Syrén, Per-Olof \\
\hline Doerr, Markus & Liolios, Konstantinos A. & Szilágyi, Istvan \\
\hline Dolzhenko, Anton V. & Lisi, Luciana & Tada, Shohei \\
\hline Domine, Marcelo Eduardo & Litinas, Konstantinos & Taddei, Maurizio \\
\hline Donat, Felix & Liu, Jinyun & Tai, Cheuk-Wai \\
\hline Dong, F. & Liu, Juewen & Tajiri, Kazuya \\
\hline Drelinkiewicz, Alicja & Liu, Xiaoxi & Takagaki, Atsushi \\
\hline $\mathrm{Du}$, Guodong & Llorca, Jordi & Takagi, Yasumasa \\
\hline Du, Xusheng & Lockridge, Oksana & Takasu, Kiyosei \\
\hline Dudding, Travis & Long, Brian & Takayuki, Ban \\
\hline Duprez, Daniel & Longo, Pasquale & Tamura, Osamu \\
\hline Dusselier, Michiel & Lonnberg, Harri & Tanaka, Shinji \\
\hline Dutta, Biswanath & Lopez, Francesco & Tanaka, Yoshiyuki \\
\hline Edalati, Kaveh & Lopez, Gartzen & Tanemura, Kiyoshi \\
\hline
\end{tabular}


Eddy, Nicholas

Egami, Hiromichi

Ehm, Christian

Eibes, G.

Emanuelsson, Emma

Emanuelsson, Emma Patterson

Engel, Paul C.

Engle, Keary

Epelde Bejerano, Eva

Escola, José María

Estrine, Boris

Evangelisti, Claudio

Faccio, Greta

Fampiou, Ioanna

Fañanas, Francisco J.

Faraldos, Marisol

Faria Albanese, Jimmy Alexander

Faria, J.

Farnood, Ramin R.

Farrauto, Robert J.

Favre-Réguillon, A.

Fechete, Ioana

Fehrmann, Rasmus

Felpin, François-Xavier

Fenning, David

Fernandes, P.

Fernandes, Pedro

Fernandez Marchante, Carmen M

Fernandez Morales, Francisco Jesus

Fernández, Roberto

Fernandez-Lafuente, Roberto

Ferraz, Ricardo

Ferri, Davide

Fettkenhauer, Christian

Fiore, Silvia

Fischer, Christin

Fischmeister, Cedric

Fishman, Ayelet

Fleischer, Ivana

Fliedel, Christophe

Flox, Cristina

Fochi, Mariafrancesca

Fokwa, Boniface
López, Moisés Canle

Lourenço, João

Lovell, Emma

$\mathrm{Lu}$, Chen

Luber, Sandra

Luh, Tien-yau

Luo, Jizhong

Luque, Rafael

Lv, Kangle

Ma, Wenping

Macario, Anastacia

Macdonald, Thomas

Mahajan, Devinder

Mahrwald, Rainer

Maireles-Torres, Pedro

Mäki-Arvela, Päivi

Makkee, Michiel

Mamane, Victor

Mancini, Ines

Manoury, Eric

Manthina, Venkata

Marchionni, Andrea

Mariangela, Longhi

Maric, Milan

Marichev, Kostiantyn

Maricruz, Sanchez-Sanchez

Marín, Pablo

Marini, Francesca

Marino, Attilio

Marr, Andrew C.

Martín, Beatriz De Rivas

Martínez, Antonio

Martínez-Arias, Arturo

Martinez-Triguero, Joaquin

Martín-Ramos, Pablo

Martins, Luísa

Martins, Rui C.

Massa, Antonio

Mastrorilli, Piero

Mateo, Cesar

Matsuda, Tomoko

Matsui, Daisuke

Mauriello, Francesco
Tao, Shanwen

Tasis, Dimitrios

Tayade, Rajesh J.

Tesser, Riccardo

Testino, Andrea

Thamaraparambil Jayaram, Dhanya

Thomas, Andrew G.

Thorimbert, Serge

Timko, Michael T.

Tischler, Dirk

Tojo, Concha

Tomaiuolo, Giovanna

Topakas, Evangelos

Torres, Tomas

Toshio, Koizumi

Tosoni, Sergio

Touraud, Didier

Toyao, Takashi

Traulsen, Marie Lund

Triantafyllidis, Kostas S.

Tricoli, Antonio

Trincone, Antonio

Trovarelli, Alessandro

Trusso Sfrazzetto, Giuseppe

Trzeciak, Anna M.

Tsai, De-Hao

Tseng, Hui-hsin

Tsubaki, Shuntaro

Tsuneda, Takao

$\mathrm{Tu}, X_{\text {in }}$

Tzounis, Lazaros

Ueda, Mitsuhiro

Upare, Pravin P.

Valente, Anabela A

Valla, Julia A.

Valladares, Luis De Los Santos

Valov, Ilia

Van Schepdael, Ann

Vannucci, Aaron K.

Vassalini, Irene

Védrine, Jacques

Velasco-Velez, Juan Jesús

Venditti, Iole 


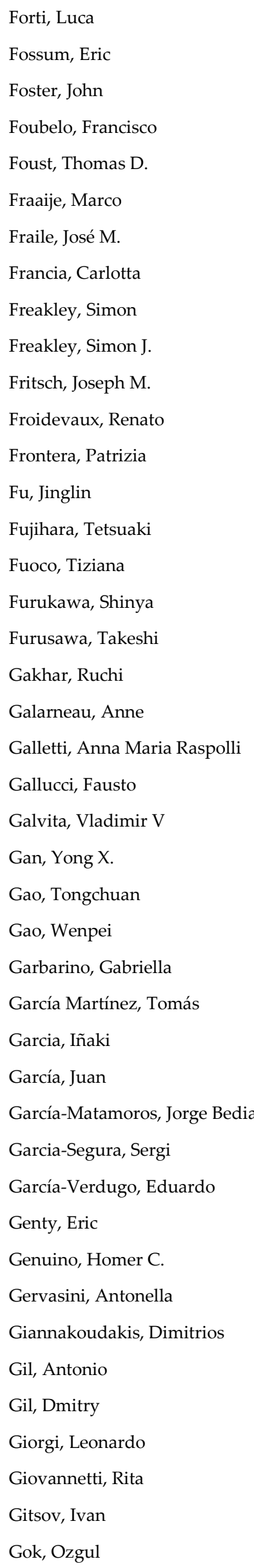

Mavila, Sudheendran

Mazzeo, Mina

Mazzoni, Rita

McCue, Alan John

Medronho, Bruno

Mehring, Michael

Mei, Bastian

Meille, Valérie

Melchionna, Michele

Melero, Juan Antonio

Milanese, Chiara

Milanesio, Marco

Milani, Dia

Millar, Graeme

Minami, Yasunori

Minegishi, Tsutomu

Mino, Lorenzo

Misaki, Tomonori

Miseki, Yugo

Miura, Tsuyoshi

Miyamoto, Manabu

Miyao, Toshihiro

Miyauchi, Masahiro

Mok, Young Sun

Moliner, Manuel

Monai, Matteo

Montalvo, Rafael

Monteverde Videla, Alessandro Hugo

Morales Guio, Carlos

Morales, Gabriel

Moreno Tost, Ramón

Moreno, Andrés

Moreno, Inés

Moreno, Jovita

Moretti, Giuliano

Mori, Kohsuke

Morita, Nobuyoshi

Mossin, Susanne

Mourdikoudis, Stefanos

Muench, Falk

Mukarakate, Calvin

Muley, Pranjali

Muller, Ulrich
Venezia, Anna Maria

Verardi, Alessandra

Vernoux, Philippe

Vicaria, José M.

Vidal, Hilario

Vigier, Karine

Vigolo, Brigitte

Villa, Alberto

Vioux, André

Virginie, Mirella

Vital, Joaquim Silvério Marques

Vitiello, Rosa

Vizcaíno, Arturo J.

Vlachos, Dimitrios

Vohs, John M.

Voiry, Damien

Wakihara, Toru

Walters, Michael

Walton, Alex

Wang, Cong

Watanabe, Hiromichi

Watanabe, Motonori

Wehinger, Gregor D.

Wickstrom, Lauren

Wills, Martin

Wohlrab, Sebastian

Wojcieszak, Robert

Wolfson, Adi

Wong, Wallace W. H.

Woolridge, Elisa

Wu, Ho-Shing

Wu, Kevin C.-W.

Wu, Ming-Chung

Wyman, Ian

Xi, Kai

Xie, Jingxiu

$\mathrm{Xu}, \mathrm{Ya}$

Xue, Xingjian

Yakura, Takayuki

Yamada, Yusuke

Yamaguchi, Aritomo

Yan, Kai

Yan, Ning 


\begin{tabular}{|c|}
\hline Gokhale, Rohan \\
\hline Gonsalvi, Luca \\
\hline Goodall, Russell \\
\hline Gossage, R. A. \\
\hline Gotor-Fernández, Vicente \\
\hline Gouany, Frady \\
\hline Gracia-Espino, Eduardo \\
\hline Grey, Carl \\
\hline Grogan, Gideon \\
\hline Grunwaldt, Jan-Dierk \\
\hline Gruttadauria, Michelangelo \\
\hline Gudiminchi, Rama \\
\hline Guerrero-Pérez, Olga \\
\hline Guidotti, Matteo \\
\hline Guijt, Rosanne M. \\
\hline Gupton, B. Frank \\
\hline Ha, Donhyung \\
\hline Ha, Kyoung-Su \\
\hline Haan, John L. \\
\hline Habibi, Biuck \\
\hline Hagiwara, Hidehisa \\
\hline Hahn, Jae Ryang \\
\hline Hallett-Tapley, Geniece \\
\hline Halpegamage, Sandamali \\
\hline Hamilton, Jeremy \\
\hline Han, Byungchan \\
\hline Han, Hyun Soo \\
\hline Han, Jaehong \\
\hline Hanaor, Dorian \\
\hline Handoko, Albertus \\
\hline Hanefeld, Ulf \\
\hline Hansen, Kent Kammer \\
\hline Haouas, Mohamed \\
\hline Hara, Kenji \\
\hline
\end{tabular}

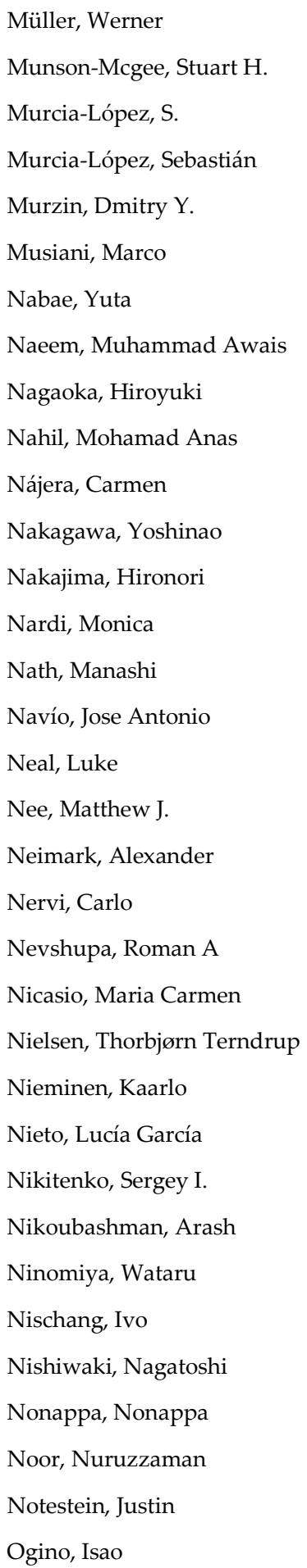

\begin{tabular}{|c|}
\hline Yan, Qimin \\
\hline Yanagida, Sayaka \\
\hline Yang, Chi-Ta \\
\hline Yang, Dong \\
\hline Yasuda, Masahide \\
\hline Yasukawa, Kiyoshi \\
\hline Yentekakis, Ioannis V. \\
\hline Yerabolu, Jayasudhan Reddy \\
\hline Yin, Shi \\
\hline Yoon, Hyun \\
\hline Yoshimoto, Makoto \\
\hline Yoshiyuki, Kuroda \\
\hline Yu, Byung Deok \\
\hline Yu, Taekyung \\
\hline Yuan, Min-Hao \\
\hline Yumoto, Isao \\
\hline Yumura, Takashi \\
\hline Yuta, Nishina \\
\hline Zetterberg, Johan \\
\hline Zhang, Jingjing \\
\hline Zhang, Miao \\
\hline Zhang, Qinghai \\
\hline Zhang, Xueqiang (Alex) \\
\hline Zhao, Di \\
\hline Zhao, Evan Wenbo \\
\hline Zhao, Zhenghang \\
\hline Zhou, Wei \\
\hline Zhu, Shan \\
\hline Zhuang, Quan \\
\hline Zhukovskyi, Maksym \\
\hline Zidki, Tomer \\
\hline Ziegler, Thomas \\
\hline Ziegler-Borowska, Marta \\
\hline Zinck, Philippe \\
\hline
\end{tabular}

(C) 2018 by the authors. Licensee MDPI, Basel, Switzerland. This article is an open access article distributed under the terms and conditions of the Creative Commons Attribution (CC BY) license (http://creativecommons.org/licenses/by/4.0/). 